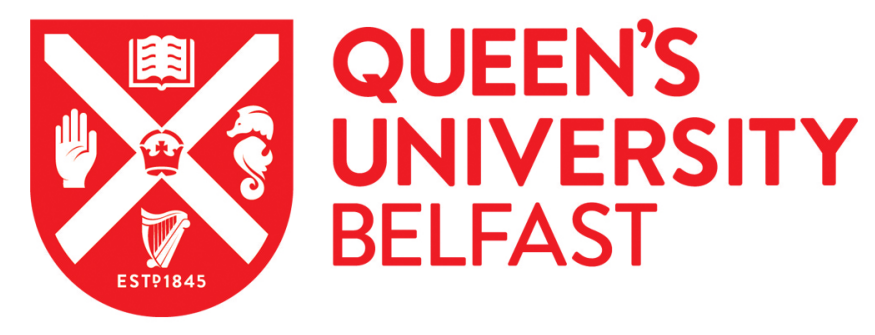

\title{
Spatially Resolved Measurements of Laser Filamentation in Long Scale Length Underdense Plasmas with and without Beam Smoothing
}

Sarri, G., Cecchetti, C., Jung, R., Hobbs, P., James, S., Lockyear, J., Stevenson, R. M., Doria, D., Hoarty, D. J., Willi, O., \& Borghesi, M. (2011). Spatially Resolved Measurements of Laser Filamentation in Long Scale Length Underdense Plasmas with and without Beam Smoothing. Physical Review Letters, 106(9), [095001]. https://doi.org/10.1103/PhysRevLett.106.095001

Published in:

Physical Review Letters

Document Version:

Publisher's PDF, also known as Version of record

Queen's University Belfast - Research Portal:

Link to publication record in Queen's University Belfast Research Portal

Publisher rights

(C) 2011 American Physical Society

\section{General rights}

Copyright for the publications made accessible via the Queen's University Belfast Research Portal is retained by the author(s) and / or other copyright owners and it is a condition of accessing these publications that users recognise and abide by the legal requirements associated with these rights.

Take down policy

The Research Portal is Queen's institutional repository that provides access to Queen's research output. Every effort has been made to ensure that content in the Research Portal does not infringe any person's rights, or applicable UK laws. If you discover content in the Research Portal that you believe breaches copyright or violates any law, please contact openaccess@qub.ac.uk. 


\title{
Spatially Resolved Measurements of Laser Filamentation in Long Scale Length Underdense Plasmas with and without Beam Smoothing
}

\author{
G. Sarri, ${ }^{1}$ C. A. Cecchetti, ${ }^{1, *}$ R. Jung, ${ }^{2}$ P. Hobbs, ${ }^{3}$ S. James,${ }^{3}$ J. Lockyear, ${ }^{3}$ R. M. Stevenson, ${ }^{3}$ D. Doria, ${ }^{1}$ D. J. Hoarty, ${ }^{3}$ \\ O. Willi, ${ }^{2}$ and M. Borghesi ${ }^{1}$ \\ ${ }^{1}$ School of Mathematics and Physics, The Queen's University of Belfast, Belfast, BT7 1NN, United Kingdom \\ ${ }^{2}$ Institute for Laser and Plasma Physics, Heinrich Heine University, Dusseldorf, Germany \\ ${ }^{3}$ AWE, Aldermaston, Reading, Berkshire RG7 4PR, United Kingdom
}

(Received 7 January 2010; published 28 February 2011)

\begin{abstract}
The onset of filamentation, following the interaction of a relatively long ( $\left.\tau_{L} \simeq 1 \mathrm{~ns}\right)$ and intense $\left(I_{L} \simeq 5 \times 10^{14} \mathrm{~W} / \mathrm{cm}^{2}\right)$ laser pulse with a neopentane filled gas bag target, has been experimentally studied via the proton radiography technique, in conditions of direct relevance to the indirect drive inertial confinement fusion scheme. The density gradients associated with filamentation onset have been spatially resolved yielding direct and unambiguous evidence of filament formation and quantitative information about the filamentation mechanism in agreement with previous theoretical modelings. Experimental data confirm that, once spatially smoothed laser beams are used, filamentation is not a relevant phenomenon during the heating laser beams propagation through typical hohlraum gas fills.
\end{abstract}

DOI: 10.1103/PhysRevLett.106.095001

PACS numbers: 52.38.Hb, 52.57.-z, 52.70.Nc

The filamentation of a laser beam as it propagates through an underdense plasma has been investigated both experimentally and theoretically over several years, [1-8] receiving recently renewed attention due to its relevance to inertial confined fusion (ICF) in both direct drive [9] and indirect drive [10] configurations. Filamentation onset in an underdense plasma is in fact a highly detrimental process since it can enhance energy losses associated to stimulated Raman scattering (SRS) and stimulated Brillouin scattering (SBS) [1]. In the indirect drive configuration, the necessity of filling the hohlraum targets with a low $Z$, low density gas [11] demands then a detailed knowledge of laser beam propagation through underdense long-scale plasmas as resulting from the ionization of the gas fill. A strong correlation between filamentation and SBS has also been demonstrated for laser-plasma interactions resembling the heating beam propagation through the coronal plasma in the direct drive ICF [12,13]. It is therefore of crucial importance to use a laser beam with an easily controllable intensity profile and this is generally achieved by inserting a random phase plate (RPP) in the laser path [14]. This device, however, even if it ensures an overall smooth envelope of the laser intensity profile, induces high-frequency, small-scale intensity speckles. Better results are obtained if RPPs are used in conjunction with additional smoothing techniques such as spectral dispersion smoothing (SSD) [15] and polarization smoothing (PS) [16]. The plasma density modulations, induced by the laser filaments seeded by such intensity speckles, have proven to be experimentally challenging to detect. This is because they have typical transverse dimensions of the order of a few micrometers (roughly given by the product between the focal number of the focusing system and the laser wavelength) and a relative amplitude of the order of
$10 \%$ [5]. Previous experimental work has thus inferred the onset of filamentation indirectly, from measurements of backscattered SBS and SRS spectra $[1,4,12,13]$ or from measurements of the laser propagation characteristics via optical interferometry $[5,6]$. However, no spatially resolved measurements of the density modulations associated with the filamentation of the laser beam have been reported in literature so far.

We present here a novel approach to the experimental study of filamentation, where the filaments are detected via the deflection of a proton probing beam, caused by the electric field associated to the density gradients across the laser filaments. This technique allowed, for the first time, to spatially resolve the plasma density modulations associated with the laser beam filaments, with and without the insertion of a RPP in the laser path, clarifying the filamentation mechanism in agreement with published analytical and numerical models.

The experiment, conducted at the HELEN laser facility [17] at AWE, was designed to investigate the filament formation using the proton radiography technique [18] when a single heating laser beam $\left(\lambda_{L}=0.527 \mu \mathrm{m}, I_{L} \simeq\right.$ $5 \times 10^{14} \mathrm{~W} / \mathrm{cm}^{2}, \tau_{L}=1 \mathrm{~ns}$ temporally flattop with raising time $\simeq 100 \mathrm{ps}$ ) irradiated gas bags filled with a neopentane $\left(\mathrm{C}_{5} \mathrm{H}_{12}\right)$ gas. The gas bag targets, similar to those routinely used in this type of experiments (on NOVA [4], NIF [19], and AWE [1]), are formed by gluing polyimide membranes to an aluminum washer $\simeq 400 \mu \mathrm{m}$ thick. When inflated, the membrane stretches to a thickness of $\simeq 350 \mathrm{~nm}$ forming an oblate gas volume with a diameter of approximately $(2.5 \pm 0.3) \mathrm{mm}$. The use of an $f / 3$ focusing lens in conjunction with a RPP produced a $250 \mu \mathrm{m}$ focal spot onto the gas bag wall. The RPP induced a smooth envelope of the laser near field profile with, superimposed, 
intensity fluctuations with a typical width of $4 \pm 1 \mu \mathrm{m}$, separation of $20 \pm 5 \mu \mathrm{m}$, and amplitude of about $1 / 3 I_{L}$. The simulated density and temperature profiles of the background plasma induced by such an interaction can be found in [1] for experimental conditions practically identical to ours. The probing proton beam was produced using the CPA [20] arm of HELEN $\left(\lambda_{L}=1.053 \mu \mathrm{m}, I_{L} \geq\right.$ $10^{19} \mathrm{~W} / \mathrm{cm}^{2}, \tau_{L}=700 \mathrm{fs}$ ). This laser pulse was focused onto a $20 \mu \mathrm{m}$ gold foil using an $f / 3$ off-axis parabola, generating, via target normal sheath acceleration [21], a directional proton beam. This proton beam after having transversely probed the interaction region, was recorded on a stack of dosimetrically calibrated radiochromic films (RCF) [22] (we refer the reader to [18] for the typical setup of this probing technique). Proton radiography allows detection of electric field distributions at the interaction plane, during a high intensity laser-plasma interaction, by measuring the spatial modulations of the proton beam $\left(\delta n_{p} / n_{p}\right)$ at the detector plane [18]:

$$
\left\langle E_{y}\right\rangle \simeq-\frac{2 \varepsilon_{p} M}{e L b} \int \frac{\delta n_{p}}{n_{p}} d y,
$$

where $M \approx 11$ is the geometrical magnification [18], $\varepsilon_{p}$ is the probing proton energy, $L \approx 3 \mathrm{~cm}$ is the distance between the target and the RCF stack, and $b$ is the longitudinal dimension of the nonzero electric field. The probe proton trajectories can, in principle, be affected also by magnetic fields in the plasma. However, Meezan et al. [1] estimate, for experimental parameters substantially identical to ours, a maximum magnetic field of $1 \mathrm{~T}$ by the end of the laser pulse; over the typical spatial scale of a filament, a magnetic field of this amplitude would induce proton deflections which are negligible compared to the one induced by the electric fields (that, as we will see in the following, are of the order of $10^{6}-10^{7} \mathrm{~V} / \mathrm{m}$ ) and can thus be neglected in Eq. (1). During the experiment, the filamentation onset was examined for different $\mathrm{C}_{5} \mathrm{H}_{12}$ pressures (i.e., 0.2, 0.5 , and 0.8 bar), with and without the insertion of a RPP in the laser path. The pressures given above correspond, once the gas is fully ionized, to electron densities of $0.05,0.13$, and 0.21 times the critical density for $0.527 \mu \mathrm{m}$ light $\left(n_{c}=4 \times 10^{21} \mathrm{~cm}^{-3}\right)$. These densities have been chosen in order to lay below, above, and at the threshold condition for filamentation onset in the case of RPP-smoothed laser beams: $\left(n_{e} / n_{c}\right)\left(v_{0}^{2} / v_{e}^{2}\right)(2 \pi f)^{2}>1$ [8]. Here $f$ is the focal number of the focusing system and $v_{0}\left(v_{e}\right)$ is the electron quiver (thermal) speed. In the ICF scenario, this condition can be more conveniently written in terms of a figure of merit $Q$ as [7]:

$$
Q=\frac{I_{L}}{10^{13} \mathrm{~W} / \mathrm{cm}^{2}}\left(\frac{\lambda_{L}}{1 \mu \mathrm{m}}\right)^{2} \frac{n_{e}}{n_{c}} \frac{3 \mathrm{keV}}{T_{e}(\mathrm{keV})}\left(\frac{f}{8}\right)^{2}>1 .
$$

Semiempirical results suggest that the additional insertion of SSD and PS devices sets this threshold to be $Q>2$ [7]. Using Eq. (2) we expect the corresponding $Q$ values to be $0.44,0.9$, and 1.5 respectively; this is concluded on the basis that calculations indicate an electron temperature in the plasma of $T_{e} \approx 0.8 \mathrm{keV}$ [1].

A representative image exemplifying the main features observable in the proton radiographs is shown in Fig. 1(a). A highly modulated, elliptical deflection pattern is present in correspondence to the impact of the laser pulse on the gas bag skin. Ahead of this, the propagation region of the laser through the plasma is visible. This is where the eventual onset of filamentation is expected to be detected [dashed square in Fig. 1(a)]. The following figures [Figs. 1(b)-1(e)] show a zoom of such region for the background densities mentioned above, with and without the insertion of a RPP in the laser path. All of these cases refer to a probing time of $100 \mathrm{ps}$ after the ns laser beam has reached its intensity plateau and a probing proton energy of $\varepsilon_{p} \approx 4 \mathrm{MeV}$. The most striking feature is the presence, only for the highest initial electron densities [Figs. 1(c) and 1 (d), i.e., when the corresponding $Q$ factor approaches or overcomes the threshold value of 1], of periodical and highly directional modulations in the proton beam, which appear to lay within the laser propagation area and with an average spatial period of about $20 \mu \mathrm{m}$. We ascribe these striations to plasma density gradients associated with laser filamentation. Removing the RPP from the laser path [Fig. 1(e)] induces these striations to be much stronger and slightly converging whereas roughly maintaining the

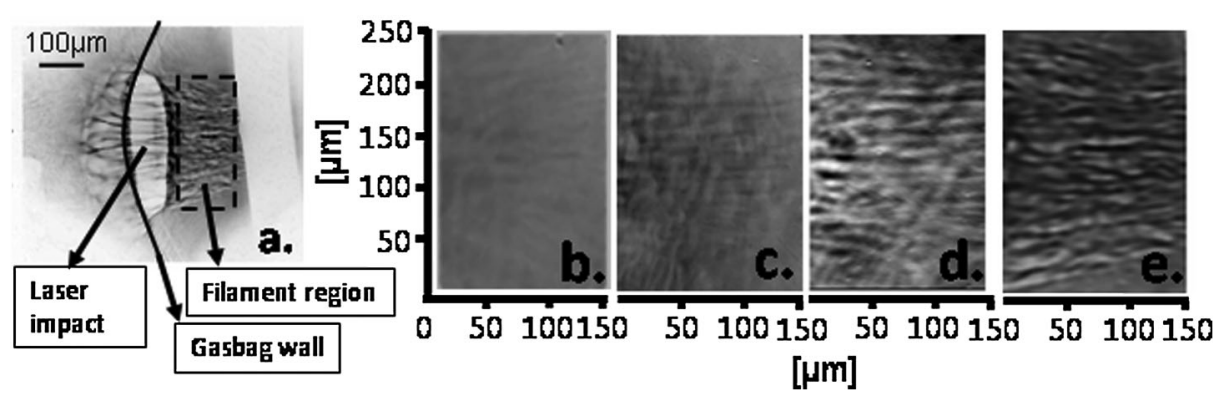

FIG. 1. (a) Typical image of a shot as it appears on the RCF layers. Zooms of the region highlighted by the dashed square in (a) for $n_{e}=0.05 n_{c}(\mathrm{~b}), n_{e}=0.13 n_{c}$ (c), $n_{e}=0.21 n_{c}(\mathrm{~d})$, and $n_{e}=0.21 n_{c}$ without RPP (e). In all the images contrast has been enhanced consistently to highlight the relevant features. 
same spatial period. This convergence can be easily understood by taking into account that the removal of the RPP induces a smaller best focus size of the laser beam. In order to maintain the same spot size and, therefore, comparable experimental conditions, the laser was slightly defocused at the gas bag wall leading to a convergent beam propagating through the plasma. The striations' amplitudes appear to increase with increasing background electron density [compare Figs. 1(c) and 1(d)]. On the other hand, they are completely absent in Fig. 1(b) $(Q=0.44)$.

It is possible to extract, directly from the RCF data, a clear correlation between the background electron density of the plasma and the filamentation spatial period for Figs. 1(c)-1(e) [see Fig. 2(a)]. The spatial period decreases with the increase of the electron density (from $\approx 25 \mu \mathrm{m}$ to $\approx 18 \mu \mathrm{m}$ ) whereas it appears to be roughly constant, within the experimental error, whether smoothing techniques are used or not.

In order to understand the real correlation between such a measured, two-dimensional, spatial modulation at the detector plane and the effective, three-dimensional, spatial distribution of the filaments within the plasma, particle tracer (PT) simulations [23] of the probing beam deflection have been performed. We used, as an input for the PT, randomly distributed filaments, with the only constraint to have a defined average mutual distance $d_{\text {fil }}$ (ranging between 10 and $40 \mu \mathrm{m}$ ). The filaments' spatial distributions were obtained with the aid of a Monte Carlo routine written for this purpose. Each filament is associated with a bipolar electrostatic field, cylindrically symmetric along the filament axis, with a diameter of $5 \mu \mathrm{m}$ and a peak amplitude of the order of $10^{7} \mathrm{~V} / \mathrm{m}$. These simulations clearly show that, for $d_{\text {fil }}=20 \mu \mathrm{m}$, the proton deflection pattern at the detector plane is still periodic [Fig. 3(a)] with a periodicity of $d_{\mathrm{PT}}=20 \pm 4 \mu \mathrm{m}$. Simulations for $d_{\mathrm{fil}}=30 \mu \mathrm{m}$ (not shown) show a similar behavior $\left(d_{\mathrm{PT}}=30 \pm 8 \mu \mathrm{m}\right)$. For $d_{\text {fil }} \approx 10 \mu \mathrm{m}$, corresponding to a densely packed filament distribution, a strongly diffused proton modulation is seen without any clear evidence of a spatial periodicity [Fig. 3(b)]; on the other hand, for $d_{\text {fil }} \geq 40 \mu \mathrm{m}$ very few filaments are present, yielding an extremely uneven proton
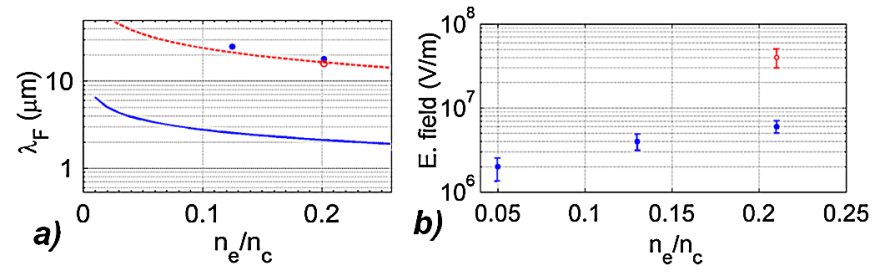

FIG. 2 (color online). (a) Comparison between the experimental spatial period of the filaments with Eqs. (3) (blue solid line) and (4) (red dashed line). (b) Modulation of the electric field across the filamented region as a function of the background electron density with (blue full circles) and without (red empty circle) the insertion of a RPP. deflection pattern (not shown). On the basis of these results it is reasonable to assume that a measured proton deflection periodicity of the order of $20 \mu \mathrm{m}$ is a realistic indication of the effective spatial period of the filaments.

This spatial period is also a clear indication of the ponderomotive nature of the filamentation. At intensities well below the relativistic regime (the laser dimensionless intensity is $a \simeq 10^{-2}$ ), filamentation is driven by two different mechanisms: thermal and ponderomotive. The density modulations and, consequently, the electric field modulations at which these two effects operate have, according to [2], typical spatial periods, respectively, given by $\lambda_{T}$ and $\lambda_{P}$ (expressed in $\mu \mathrm{m}$ ):

$$
\begin{gathered}
\lambda_{T}=15 \frac{\varepsilon^{3 / 16} T_{e}^{7 / 8}(1+1 / Z)^{1 / 4} \lambda_{L}^{1 / 2} \phi^{3 / 8}}{(\ln \Lambda)^{1 / 4} Z^{1 / 2} I_{L}^{3 / 8}}\left(\frac{n_{c}}{n_{e}}\right)^{5 / 8} \\
\lambda_{P}=10.4 \frac{\varepsilon^{1 / 4} T_{e}^{1 / 2}(1+1 / Z)^{1 / 2}}{I_{L}^{1 / 2}}\left(\frac{n_{c}}{n_{e}}\right)^{1 / 2}
\end{gathered}
$$

where $\left[T_{e}\right]=\mathrm{keV},\left[\lambda_{L}\right]=\mu \mathrm{m},\left[I_{L}\right]=\mathrm{W} / \mathrm{cm}^{2}$ in units of $10^{14}, \varepsilon=1-n_{e} / n_{c}, \phi=(Z+0.24) /(1+0.24 Z), \ln \Lambda$ is the Coulomb logarithm and $Z$ represents the ionization stage. In Fig. 2(a) these two theoretical curves are shown compared with the experimental data. For the electron densities used in this experiment thermal effects should induce density modulations with a spatial period of the order of 1-2 $\mu \mathrm{m}$ while ponderomotive filamentation should grow with a spatial period of the order of 10-20 $\mu \mathrm{m}$. The significant difference between these two effects allows arguing that the filamentation observed is driven by the ponderomotive force. The collisionless nature of the plasma makes in fact electron Landau damping much more effective than thermal diffusion [8], theoretically explaining the predominance of ponderomotive effects in the filamentation onset. The measured periodicity of the filaments is therefore the one that maximizes the growth rate of ponderomotive filamentation and it is thus not surprising to detect a similar periodicity whether smoothing techniques are used or not.

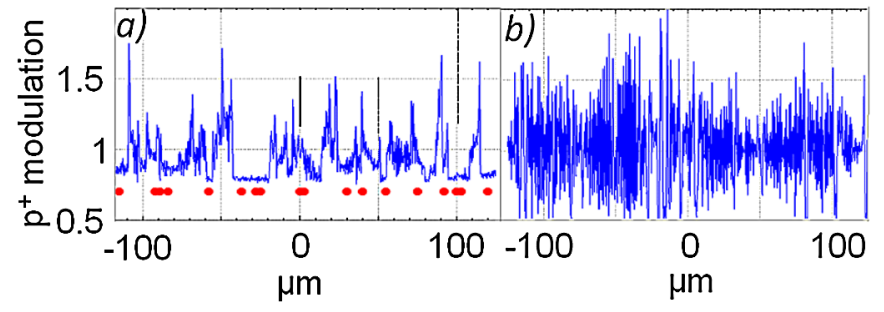

FIG. 3 (color online). Simulated deflection pattern of the probing proton beam traversing a region of randomly distributed filaments with an average period of $20 \mu \mathrm{m}$ (a) and $10 \mu \mathrm{m}$ (b). In the first frame red dots represent the projection of the filament position. 
For all the images shown in Fig. 2, the electric field distribution in the gas bags across the laser propagation axis has been extracted using Eq. (1). In all the cases, the electric field distribution, associated to pressure gradients in the filaments, is modulated and the average amplitude modulations obtained are shown in Fig. 2(b) as a function of the background electron density; the electric field modulation increases as a function of the electron density from a background level of $\approx 2 \times 10^{6} \mathrm{~V} / \mathrm{m}$ up to $\approx 6 \times 10^{6} \mathrm{~V} / \mathrm{m}$. Within the same experimental conditions, the removal of the RPP induces a jump in the electric field modulation of almost an order of magnitude (up to $\approx 4 \times 10^{7} \mathrm{~V} / \mathrm{m}$ ), proving a significant enhancement of filamentation growth in this regime. Ponderomotive filamentation is indeed expected to exhibit a higher growth rate if no smoothing techniques are used [3]. For the laser and plasma parameters used in these experiments, the ponderomotive filamentation growth rate for an unsmoothed beam is $10^{3}$ times higher than the one related to a RPP-smoothed beam explaining the stronger electric field modulation detected [see Fig. 2(b)]. These electric fields are mostly related to the plasma density gradients induced by the ponderomotive force and can be expressed as $\vec{E}_{\mathrm{fil}}=-\nabla p_{e} /\left(e n_{e}\right)$ where $\nabla p_{e}$ denotes the plasma pressure gradient. Assuming the electrons as an ideal gas $\left(p_{e}=n_{e} K_{B} T_{e}\right)$, neglecting thermal effects $\left(\nabla T_{e}=0\right)$, and approximating $\nabla n_{e} \approx \delta n_{e} / \rho_{\mathrm{fil}}\left(\rho_{\mathrm{fil}} \approx 2.5 \mu \mathrm{m}\right.$ denotes the filament radius), this estimate leads to $E_{\mathrm{fil}} \approx$ $10^{7} \mathrm{~V} / \mathrm{m}$, in good agreement with the experimental findings. This is concluded on the basis that $\delta n_{e} / n_{e}$ can be theoretically inferred to be $\approx 4 \%$ [see Eq. (23) in [24] ].

This set of experimental data is of particular interest for the indirect drive ICF scenario. Current schemes for indirect drive ICF employ hohlraum gas fills with electron densities in the range $0.05-0.15 n_{c}$ [10]. Analytical [8] models predict that filamentation instability is not expected to be excited at these electron densities, if the laser is spatially smoothed by the insertion of a RPP. Moreover numerical results and theoretical interpretation reported in [1] suggest that, for $0.05 n_{c} \leq n_{e} \leq 0.15 n_{c}$, the simulated SRS and SBS spectra can be explained without invoking filamentation of the laser beam. Indeed, our results show that at densities within this range no significant filamentation has occurred yet whereas it becomes a relevant phenomenon only for higher electron densities. This is also consistent with the filamentation threshold given by Dewald and collaborators [7] [see Eq. (2)].

In conclusion, the first spatially resolved measurements of filamentary structures in long-scale underdense plasmas are reported. Direct measurements of the electrostatic field distribution, associated with the plasma density gradients, have been performed validating previous theoretical and experimental findings. The experimental results indicate that filamentation is not a relevant phenomenon during the propagation of the heating laser beams through the hohlraum gas fill in recently proposed indirect drive ICF schemes.

Funding for this research has been provided by the AWE Academic Access Scheme, EPSRC Grants No. EP/ E035728/1 and No. EP/C003586/1, and by DFG TR 18, GK 1203. We also acknowledge the support of the HELEN Facility laser and target preparation personnel.

*Present address: IPCF, Consiglio Nazionale delle Ricerche, CNR campus, Pisa, Italy.

[1] N. B. Meezan et al., Phys. Plasmas 11, 5573 (2004).

[2] E. M. Epperlein, Phys. Rev. Lett. 65, 2145 (1990).

[3] A. J. Schmitt, Phys. Fluids 31, 3079 (1988).

[4] J. D. Moody et al., J. Fusion Energy 12, 323 (1993).

[5] P. E. Young, Phys. Plasmas 2, 2825 (1995).

[6] S. Wilks et al., Phys. Rev. Lett. 73, 2994 (1994).

[7] E. L. Dewald et al., Plasma Phys. Controlled Fusion 47, B405 (2005).

[8] R. L. Berger et al., Phys. Fluids B 5, 2243 (1993).

[9] R. L. McCrory et al., Nucl. Fusion 41, 1413 (2001).

[10] J. Lindl et al., Phys. Plasmas 11, 339 (2004).

[11] R. L. Kauffman et al., Phys. Plasmas 5, 1927 (1998).

[12] T. Afshar-Rad et al., Phys. Rev. Lett. 76, 3242(E) (1996).

[13] S. Regan et al., Phys. Plasmas 6, 2072 (1999).

[14] Y. Kato et al., Phys. Rev. Lett. 53, 1057 (1984).

[15] S. Skupsky et al., J. Appl. Phys. 66, 3456 (1989).

[16] L. Divol et al., Phys. Rev. Lett. 100, 255001 (2008).

[17] M. J. Norman et al., Appl. Opt. 41, 3497 (2002).

[18] G. Sarri et al., New J. Phys. 12, 045006 (2010).

[19] R. L. McCrory, Nucl. Fusion 44, S123 (2004).

[20] G. A. Mourou et al., Rev. Mod. Phys. 78, 309 (2006).

[21] R. A. Snavely et al., Phys. Rev. Lett. 85, 2945 (2000).

[22] J. F. Dempsey et al., Med. Phys. 27, 2462 (2000).

[23] L. Romagnani, Ph.D. thesis, Queen's Univiersity of Belfast, U.K., 2005.

[24] V. B. Krapchev, Phys. Rev. Lett. 42, 497 (1979). 